\title{
Clinical judgment of nurses: Gravity of symptom configurations, quantity of symptoms, and extraneous variables
}

\author{
ANTHONY L. ROSSI, JR. and JOSEPH M. MADDEN \\ Rensselaer Polytechnic Institute, Troy, New York 12181
}

\begin{abstract}
Nurses' diagnostic decision making is discussed in terms of its importance and the lack of empirical research to determine training needs to prepare nurses for this function. Results of this investigation suggest that nurses base their diagnostic decisions on the number of symptoms present, independent of symptom gravity. Results also suggest that nurses are influenced by extraneous symptoms to various degrees, depending on the gravity or weakness of symptoms. It is concluded that formal training in diagnostic decision making should be an important part of nurses' preparation.
\end{abstract}

One of the most important functions of the professional nurse's role, that of making inferences regarding the state of the patient, has undergone expansion in the last two decades. This relatively recent development is at least partially the result of Lesnik's (1955) milestone study involving case decisions of judicial review that indicated that the professional nurse is legally responsible for making diagnoses. This is not difficult to understand when one considers that, although the physician is recognized as the chief diagnostician of the medical team, once his patient is admitted to the medical ward for extended observation and treatment, some of the physician's diagnostic and, therefore legal, responsibilities devolve upon the nurse. As a result of Lesnik's study, the making of a nursing diagnosis was explicitly recognized as a legal function of the nurse, as well as an independent and medically essential one.

Another factor that has assisted in making the nurse's function as a decision maker explicit has involved a broadening of the nursing profession's concept of the professional nurse. Whereas the traditional model gave little formal recognition to the cognitive aspects of the nurse's task, the recent trend in nursing is to foster an appreciation of the cognitive aspects of the nurse's role. According to Hammond (1966), "it has been traditional for nursing to emphasize perceptual and motor skills rather than cognitive skills; nurses have been primarily focused on seeing and doing rather than thinking. Modern nursing, however, emphasizes cognitive skills as well as perceptual and motor skills" (p. 134).

Although the significance of training nurses in order that they might attain some reasonable level of proficiency at conducting nursing diagnoses has been recognized by the nursing profession, attempts at delineating the nurse's cognitive task have, for the most part, revolved around determining where the nursing diagnosis leaves off and the medical diagnosis, which is the sole responsibility of the physician, begins. The central issue here has concerned the level of authority delegated the nurse in inferring the gravity of the patient's state, as well as her or his role in determining the nature of the patient's malady. Since the latter function, which concerns identification of the disease, has been recognized as being within the realm of medical diagnosis, the nurse has been prohibited from participating in such decisions. However, even though the nurse cannot participate in conducting medical diagnoses, Murchison and Nichols (1970) have stated that "the nurse is properly allowed the responsibility of judging the gravity of symptoms without engaging in medical diagnosis." Thus, although the nurse does not have the authority to conduct medical diagnoses (i.e., identify diseases via inductive inference), she/he is responsible for judging the gravity of symptoms manifested without infringing on the physician's medical diagnosis.

Unfortunately, the literature contains no empirical attempts to examine how symptom configurations of differential gravity are assimilated by the nurse in an attempt to decide what ameliorative action (i.e., administer medication, provide psychological support, notify the physician, etc.) should be initiated in response to the patient's needs. Although there seems to be general agreement on the part of those in the nursing and medical professions concerning the specific action patterns that the nurse should engage in, research regarding the cognitive antecedents of these patterns is conspicuously lacking. The purpose of this paper is to investigate the nature of such antecedents.

Since, as we have already mentioned, one of the nurse's most important functions is to make inferences regarding the gravity of sympton configurations, it seems that a reasonable first step in examining the inferential task of the nurse should entail an examination of how nurses react to symptom configurations of differential gravity. Serious symptoms should be more likely to 
stimulate action on the nurse's part than minor ones. Our first hypothesis, then, is that nurses encountering symptom configurations that are perceived as being more serious in nature would be more likely to take action than nurses who encounter configurations that are perceived as being less serious in nature.

Since any specific malady may manifest myriad symptoms via the patient's signal-sending system, we might go a step further and investigate how configurations that differ in the quantity of symptoms they contain affect the nurse's inferential process. Thus, our second hypothesis is that the nurse's decision to initiate a specific action pattern is a positive function of the number of symptoms manifested by the patient's condition. The logic behind this hypothesis is derived from the fact that, although the nurse has had a certain amount of training at identifying and evaluating symptoms that characterize a disease, the assimilation of increasingly complex symptom configurations surpasses the realm of her/his expertise. As the nurse encounters symptom configurations that require a great deal of integrative skill, it seems plausible to assume that she/he would tend to seek the assistance of the physician, whose medical training has prepared her/him to handle such complex cognitive tasks.

An interesting study by Davitz and Pendleton (1969) showed that extraneous variables influenced nurses' inferences of patient suffering. Specifically, this study showed that nurses consistently inferred greater suffering in youthful patients than in aged patients, and upper class patients were perceived to suffer significantly less than were middle or lower class patients. The Davitz and Pendleton findings concerning extraneous information seem to have important implications for the study of nursing inferences. Therefore, the present study will expand upon such implications via the inclusion of extraneous variables in symptom configurations presented to nurses for decisions. Before formulating the third hypothesis, however, it must be recognized that such extraneous variables do not occur in a vacuum. Instead, they occur in the midst of symptom configurations that the patient manifests, and such configurations differ with respect to their diagnostic utility. Whereas certain symptom profiles characteristic of a particular malady leave little doubt regarding the severity of the state of the patient, others are weaker and less important in nature, yielding a more uncertain picture. It seems reasonable to predict that extraneous variables would have a greater effect with weak symptom profiles than with strong ones. Our third hypothesis, then, is that nurses are influenced by extraneous variables and that such influence increases as the diagnostic power of physiological variables decreases.

\section{METHOD}

In order to obtain physiological symptoms, a comprehensive clinical nursing text (McCutcheon, 1970) was examined to find a malady with easily understood symptoms. The text's uremia description was selected along with 16 uremia symptoms. Thirty-two professional nurses from two nonteaching hospitals were given the 16 symptoms and asked to rank them according to their perceived diagnostic utility by placing a " 1 " next to the symptom that was most useful down to a "16" for the symptom that was perceived to be of least utility in diagnosing uremia. Table 1 gives the results.

The four symptoms with the lowest mean ranks were grouped to produce a single mean and standard deviation for this physiologically strong symptom configuration. The same was done with the four symptoms with the highest mean ranks (weak symptom configuration). A $t$ test of the difference between the means of these physiologically weak and strong groups (see Table 1) showed that the respective symptom groupings differed significantly in diagnostic utility $(t=11.4$, $\mathrm{p}=.005$ ).

Although the literature contains some cues as to the nature of extraneous information that might have an effect on the nurse's decision process, it is skimpy (e.g., Davitz \& Pendleton, 1969). Thus individual interviews with professional nurses were necessary to learn more about potentially influential extraneous variables. Fifteen professional nurses were asked if they felt the average professional nurse was capable of being at all affected by information of an extraneous nature (i.e., information aside from medically relevant data) as she or he made decisions regarding the state of the patient. If the subject's response was in the affirmative (as they all were), she or he was asked to give examples of how extraneous information might be utilized. As the nurse-subjects gave examples, the interviewer made note of the nature of each extraneous variable mentioned. Each interview was approximately $20 \mathrm{~min}$ in duration.

In order to select the more powerful extraneous variables from the 11 yielded by the interviews, an experiment was conducted to determine which of the 11 had the most powerful effect on the decision making process of the nurse. Subjects were 72 professional nurses from nearby nonteaching hospitals.

Two extremes of each of the 11 extraneous variables were formed on the basis of suggestions made by the nurses interviewed. For instance, for the variable "ward demeanor," one category was "demanding," the other, "nondemanding." Eleven scenarios were then written, one for each extraneous variable, describing an ill patient whose condition was suddenly aggravated (e.g., a patient recovering from abdominal surgery complains of sudden extreme pain). Two forms of each scenario were then prepared, with the only difference being the use of one extreme or the other of the extraneous variable.

A 5-point scale was used by the nurse-subjects to respond to the question, "Do you feel a physician should be notified for this patient?" The values of this rating scale ranged from 1 (yes)

Table 1

Results of Symptom Ranking Task

\begin{tabular}{lcclrc}
\hline Strong Symptoms & Mean Rank & SD & Weak Symptoms & Mean Rank & SD \\
\hline Urine Output Low & 3.50 & 2.59 & Ammonia Breath & 9.25 & 4.28 \\
Generalized Edema & 4.66 & 2.35 & Itchy Skin & 11.08 & 3.52 \\
Convulsion & 7.83 & 6.50 & Anemia & 11.83 & 3.12 \\
Dehydration & 7.83 & 3.27 & Oral Ulcers & 12.17 & 3.53 \\
Overall & 5.95 & 4.03 & Overall & 11.08 & 3.63 \\
\hline
\end{tabular}


to 5 (no). The "call physician" criterion was chosen on the basis of research done by Hammond, Kelly, Schneider, and Vancini (1966), who found that one of the nurse's important response possibilities when responding to an ill patient concerns notifying a physician.

Means, standard deviations, and $95 \%$ confidence limits were obtained for each extreme of each variable. This enabled comparison of the two extremes of each extraneous variable to determine if the confidence intervals of the two extremes overlapped. If they did not, it was taken as evidence that that extraneous variable does have an effect on the nurse's decision making process.

The results show that two of the extraneous variables elicited in the interviews influence the way a nurse reaches a decision. Since the confidence intervals of the "time at the hospital" variable (Week 1 vs. Week 5) were not overlapping, it was concluded that this extraneous variable did indeed influence the decision process of the nurse-subjects. The same conclusion was made regarding the variable "ward demeanor." Socioeconomic class, temperament, intelligence, personal cleanliness, physiognamy, political orientation, sex, number of roles, and age all had overlapping confidence intervals and were discarded.

In order to generate data for testing of the hypotheses, a matrix consisting of six dichotomous pieces of patient information (four physiologically relevant symptoms and two extraneous variables) was prepared. The extraneous variable "time at the hospital" was dichotomized according to whether the patient was present at the institution for either a short time, designated in the matrix as an " $\mathrm{S}$," or a long time, designated as an "L." The remaining five variables were dichotomized according to their occurrence, present $(\mathrm{Y})$ vs. not present $(\mathrm{N})$. A matrix of six pieces of patient information dichotomized in this manner yields 64 patient profiles. Taken together, these 64 profiles represented every possible combination of the dichotomized variables. The dependent variable was the respondent's decision concerning the notification of a physician.

To facilitate an examination of the effect extraneous variables have on the nurse's decision making process in the context of both the most and least important physiological cue configurations, two forms of the patient profile matrix were developed. One form included the four most important physiological symptoms of uremia along with the two extraneous variables "time at the hospital" and "ward demeanor." The second form of the patient profile matrix consisted of the four physiological symptoms of uremia that were ranked lowest, along with the same two extraneous variables that were in the first form. The two groups of predictors contained in each form appear in Table 2.

\section{RESULTS}

The results of an analysis of variance are presented in Table 3. Examination of these results indicates that

Table 2

Predictors Included in Each form

\begin{tabular}{ll}
\hline \multicolumn{1}{c}{ Form A } & \multicolumn{1}{c}{ Form B } \\
\hline Urine Output Low & Ammonia Breath \\
Generalized Edema & Itchy Skin \\
Convulsion & Anemia \\
Dehydration & Oral Ulcers \\
Ward Demeanor & Ward Demeanor \\
Time at Hospital & Time at Hospital \\
\hline
\end{tabular}

Note-Form $A=$ physiologically important symptoms; Form $B=$ physiologically weak symptoms.
Table 3

Analysis of Variance Table for Number and Strength of Symptoms

\begin{tabular}{lrrrc}
\hline & Subjects & df & MSe & F \\
\hline Strength & 156.9 & 1 & 156.9 & 1.60 \\
Number & $4,479.9$ & 6 & 746.6 & $7.53^{*}$ \\
Strength by Number & 89.4 & 6 & 14.9 & .15 \\
Within Cells & $13,385.5$ & 135 & 99.1 & \\
& $18,111.7$ & 148 & & \\
\hline
\end{tabular}

${ }^{*} p<.01$.

the nurses in this study based their responses to the 64 patient profiles on the number of symptoms present. The obtained $F$ ratio of 7.53 was significant at the .01 level. Quite surprisingly, symptom gravity (or strength) did not seem to contribute to the diagnostic judgment of the nurses in this study, as the obtained F ratio of 1.6 indicates. The lack of a significant interaction between symptom gravity and quantity is apparent from the obtained strength by number $F$ ratio of .15 .

A multiple linear regression equation was computed for each condition using the nurse's responses as the dependent variable and the six symptoms as predictors. Then, an $\mathrm{F}$ test of the significance of the contribution of the extraneous information to the total $R^{2}$ for each condition was made. The results of these tests are in Table 4. They indicate that the extraneous information, namely time at the hospital and ward demeanor, did indeed affect the way the nurses responded to the "call physician" criterion. The fact that the amount of variance accounted for by the extraneous variables combined with weak symptoms is more than twice that when combined with strong symptoms indicates that the weaker the physiological information, the greater the effect of the extraneous information.

These data shed some light on the way nurses utilize diagnostic information to make decisions concerning their patients. Murchison and Nichols (1970), as was mentioned earlier, stated that the professional nurse is responsible for taking the gravity of the patient's symptoms into account as she/he attempts to make a nursing diagnosis. The finding that nurses depend on symptom quantity and not symptom gravity to decide whether to initiate an action pattern may be a matter of concern.

An examination of the effect of extraneous information on the clinician's decisions has been long overdue. Our findings supported the hypothesis that extraneous information does come into play as at least one type of clinician, the nurse, attempts to deal with clinical information. Perhaps it is an even more important finding that the weaker the symptom configuration of the malady, the more powerful the effect of the extraneous information on the nurse's decision. This finding may have implications, not only for nurse training, but also for the psychology of human and 
Table 4

Total $\mathbf{R}^{2}$ for All Variables, for Physiologically Strong and Weak Symptoms, and F Ratios for the Additional

Contribution of the Extraneous Information

\begin{tabular}{lccccc}
\hline $\begin{array}{l}\text { Symptom } \\
\text { Strength }\end{array}$ & $\mathrm{n}$ & $\mathrm{R}^{2}$ & $\begin{array}{c}\text { Physio- } \\
\text { logical }\end{array}$ & $\begin{array}{c}\text { Extran- } \\
\text { eous }\end{array}$ & \multicolumn{1}{c}{$\mathrm{F}$} \\
\hline Strong & 96 & .696 & .661 & .029 & $8.87^{*}$ \\
Weak & 93 & .615 & .544 & .072 & $11.91^{*}$ \\
\hline
\end{tabular}

${ }^{*} p<.01$.

clinical inference in general. It suggests that the more vague and ambiguous the relevant cues become, the greater the influence of factors that, albeit adjacent to the task of interest, are irrelevant to its proper execution.

With the expansion of the nurse's role to include complex cognitive functions such as diagnosis, it is inevitable that the nursing profession will soon be faced with the decision of whether the problem solving process of the nurse is to continue being regarded as an intuitive, artistic venture or a skill acquired through rigorous training based on empirical research.

\section{REFERENCES}

Davitz, L. J., \& Pendelton, S. Nurses' inferences of suffering. Nursing Research, 1969, 18, 100-107.

Hammond, K. R. Clinical inference in nursing: A psychologist's viewpoint. Nursing Research, 1966, 15, 27-38.

Hammond, K. R., Kelly, K., Schneider, R. J., \& Vancini, M. Clinical inference in nursing: Analyzing cognitive tasks representative of nursing problems. Nursing Research, 1966, 15, 134-138.

Lesnik, M. J. Nursing practice and the law. Philadelphia: Lippincott, 1955.

McCutcheon, M. Care of the patient with common medicalsurgical disorders: A textbook for nurses. New York: McGrawHill, 1970.

Murchison, I., \& Nichols, T. Legal foundations of nursing practice. London: McMillan, 1970.

(Received for publication July 10, 1979.) 